\title{
Bi-level decision-making approach for planning electric power systems under stochastic-interval-parameter uncertainty
}

\author{
Yizhong Chen ${ }^{\mathrm{a}}$, $\mathrm{Li} \mathrm{He}^{\mathrm{b}}, \mathrm{Xi}$ Cheng ${ }^{\mathrm{C}}$, Jing $\mathrm{Li}^{\mathrm{d}}$ and Hongwei $\mathrm{Lu}^{\mathrm{e}}$ \\ Renewable Energy Academy, North China Electric Power University,Beijing, 102206, China \\ afjchenyizhong@163.com, ${ }^{\mathrm{b}} \mathrm{li}$.he@ncepu.edu.cn, ${ }^{\mathrm{C}} \mathrm{qm}$ chengxi@163.com, ${ }^{\mathrm{d}}$ lijingb10@163.com, ${ }^{\mathrm{P}} \mathrm{luh}$ \\ w@ncepu.edu.cn
}

Keywords:Bi-level programming, electric power systems, uncertainty, stochastic.

\begin{abstract}
This study develops an inexact stochastic bi-level programming model (ISBPM) for planning electric power systems (EPS) under uncertainty. ISBPM is formulated based on interval-parameter programming (IPP), stochastic mathematical programming (SMP) and bi-level linear programming (BLP). In the ISBPM framework, IPP and SMP are useful in dealing with parameters expressed as interval and stochastic values, and BLP can address the Leader-Follower problem with environmental target at the upper-level and economic target at the lower-level. Then, the developed model is applied to an actual case of EPS in Fengtai District, Beijing, China. Results can provide non-compromised schemes with consideration preferences of the two decision-makers. The integrated targets of economy and environment would be also achieved.
\end{abstract}

\section{Introduction}

Growing electricity demand and rising concerns over air pollution have driven the decision-makers to contemplate comprehensive planning in electric power systems (EPS). Previously, there issues were usually solved synchronously based on numerous mathematical techniques. While in practice, air pollution control and electricity generation management are placed at different decision-making levels, wherein air pollution control is requested by the environment sector and electricity generation management is more inclined to follow local authority's habits and behaviors. Obviously, the problem of planning EPS can be presented as a Leader-Follower game. Neither single-objective nor multi-objective approaches can reflect the above issues. However, it is suitable to address the problem with bi-level programming model ${ }^{[1]}$.

Nevertheless, multiple uncertainties have long challenged decision-makers, especially stochastic and interval parameters. Therefore, an inexact stochastic bi-level programming model (ISBPM) is developed in this study for planning regional EPS, which is based on interval-parameter programming (IPP), stochastic mathematical programming (SMP) and bi-level linear programming (BLP). Then, a case study on an actual EPS in the district of Fengtai, Beijing, China is performed to demonstrate the application and effectiveness of the ISBPM. Actually, the problems of electricity consumption and air quality in the study area are becoming intensified.

\section{Mathematical models}

A general ISBPM can be formulated as follows:

[Upper level]:

$$
\text { Min } \quad A P E^{ \pm}=\sum_{r=1}^{6} \sum_{g=1}^{3} \sum_{t=1}^{3} X E_{r, t}^{ \pm} \cdot \eta_{r, g, t}^{ \pm}
$$

Subject to:

$\sum_{r=1}^{6} X E_{r, t}^{ \pm} \cdot \eta_{r, g, t}^{ \pm} \leq T A P_{g, t}^{ \pm}, \forall g, t(2)$

[Lower level]: 
Min

$$
\begin{aligned}
\operatorname{COST}^{ \pm}= & \sum_{j=1}^{2} \sum_{t=1}^{3} P_{j, t}^{ \pm} \cdot X_{j, t}^{ \pm}+\sum_{t=1}^{3} P I_{t}^{ \pm} \cdot X R_{t}^{ \pm}+\sum_{r=1}^{6} \sum_{t=1}^{3} P E_{r, t}^{ \pm} \cdot X E_{r, t}^{ \pm} \\
+ & \sum_{r=1}^{6} \sum_{t=1}^{3}\left(P F_{r, t}^{ \pm} \cdot Z_{r, t}^{ \pm}+P V_{r, t}^{ \pm} \cdot X C_{r, t}^{ \pm}\right)+\sum_{r=1}^{6} \sum_{g=1}^{3} \sum_{t=1}^{3} X E_{r, t}^{ \pm} \cdot \\
& \eta_{r, g, t}^{ \pm} \cdot P T_{r, g, t}^{ \pm}+\sum_{r=1}^{6} \sum_{t=1}^{3} X E_{r, t}^{ \pm} \cdot S C_{r, t}^{ \pm} \cdot P C^{ \pm} t
\end{aligned}
$$

Subject to:

$$
\begin{gathered}
\sum_{r=1}^{2} X E_{r, t}^{ \pm} \cdot F E_{r, t}^{ \pm} \leq X_{1, t}^{ \pm}, \forall t \\
X E_{6, t}^{ \pm} \cdot F E_{6, t}^{ \pm} \leq X_{2, t}^{ \pm}, \forall t \\
X E_{r, t}^{ \pm} \cdot F E_{r, t}^{ \pm} \leq U P_{r, t}^{ \pm(P i)}, \forall r=3,4,5 ; t \\
Z_{r, t}=\left\{\begin{array}{ll}
1 & \text { if expansion is undertaken, } \\
0 & \text { if otherwise, }
\end{array} \forall r, t\right. \\
X C_{r, t}^{ \pm} \leq X C_{r, t, \max }^{ \pm}, \forall r, t(8) \\
X E_{r, t}^{ \pm} \geq 0, \quad \forall r, t \quad X_{j, t}^{ \pm} \geq 0, \quad \forall j, t
\end{gathered}
$$

Where superscript “ \pm ” represents interval parameters; $r$ is index for electricity conversion technology, $r=1,2 \ldots$ 6. $r=1$ for natural gas-fired (NF), 2 for distributed-power (DP), 3 for solar-power (SP), 4 for wind-power (WP), 5 for geothermal-power (GP), 6 for biomass-power (BP); $t$ is index for planning period, $t=1$, 2, 3. $t=1$ for 2016-2020, 2 for 2021-2025, 3 for 2026-2030; $j$ is index for energy carries, $j=1,2$. $j=1$ for natural gas, 2 for biomass; $g$ is index for air pollution, $g=1,2,3 . g=1$ for $\mathrm{SO}_{2}, 2$ for $\mathrm{NO}_{\mathrm{x}}, 3$ for $\mathrm{PM}_{10}$; $A P E$ represents total air pollution emissions (tonne); $X E_{r, t}$ represents electricity generation from conversion technology $r$ during period $t(\mathrm{GW} \mathrm{h}) ; \eta_{r, g, t}$ represents the amount of air pollution $g$ emission from conversion technology $r$ during period $t$ (tonne/GW h); $T A P_{g, t}$ represents the allowable emission of air pollution $g$ during period $t$ (tonne); COST represents system cost (RMB $¥) ; P_{j, t}$ represents cost for energy carry $j$ during period $t$ (RMB $¥ / \mathrm{TJ}$ ); $X_{j, t}$ represents the amount of energy carry $j$ during period $t(\mathrm{TJ})$; $P I_{t}$ represents cost for imported electricity during period $t(\mathrm{RMB} ¥ / \mathrm{GW} \mathrm{h}) ; X R_{t}$ represents the amount of imported electricity during period $t(\mathrm{RMB} ¥ / \mathrm{GW} \mathrm{h}) ; P E_{r, t}$ represents cost for conversion technology $r$ during period $t$ (RMB $¥ / G W \mathrm{~h}) ; P E_{r, t}$ and $P V_{r, t}$ represent cost for the fixed-charge and variable expansion of conversion technology $r$ during period $t$, respectively (RMB $¥ / G W$ ); $Z_{r, t}$ represents binary variable for conversion technology $r$ during period $t ; P T_{r, g, t}$ represents of treatment cost of air pollution $g$ from conversion technology $r$ during period $t(\mathrm{RMB} ¥ / \mathrm{GW} \mathrm{h})$; $S C_{r, t}$ represents the $\mathrm{CO}_{2}$-emission from conversion technology $r$ during period $t$ (tonne/GW h); $P C_{t}$ represents per carbon tax during period $t$ (RMB ¥/GW h); $F E_{r, t}$ represents unit of power generation for conversion technology $r$ during period $t$ $(\mathrm{TJ} / \mathrm{GW} \mathrm{h}) ; X C_{r, t}$ and $X C_{r, t, \max }$ represent capacity expansion and maximum capacity expansion for conversion technology $r$ during period $t$, respectively(GW); $U P_{r, t}$ represents availabilities of renewable energy resources for electricity generation in period $t(\mathrm{TJ}) ; \mathrm{p}_{\mathrm{i}}$ represents violation level, which corresponds to the availabilities of renewable energy resources.

\section{Results analysis}

\subsection{Electricity generation pattern}

Through solving the ISBPM, solutions of electricity generation can be obtained (shown in Fig.1). Three violation levels (i.e. $\mathrm{p}_{\mathrm{i}}=0.01,0.05$ and 0.1 ) are taken into consideration. Results show that the amount of natural gas-fired would fall steadily from $\mathrm{p}_{\mathrm{i}}=0.01$ to $\mathrm{p}_{\mathrm{i}}=0.1$, with total generation being [7989.41, 10502.99], [7832.07, 10321.44] and [7662.98, 9851.90] GW h under $\mathrm{p}_{\mathrm{i}}=0.01,0.05$ and 0.1 , respectively. The changes in biomass-power and geothermal-power are not obvious under different violation levels, but their contributions increase with the time. Take $\mathrm{p}_{\mathrm{i}}=0.01$ as example, electricity provided by biomass-power would increase from $[1500.00,1680.00] \mathrm{GW}$ h in period 1 to [3300.00, 
3420.00] GW h in period 3, and that provided by geothermal-power would increase from [102.38, 143.02] GW h in period 1 to [106.17, 148.19] GW h in period 3. However, there are marked variations in solar-power and wind-power under different violation levels. For example, contributions from solar-power and wind-power over the planning horizon would increase from [1476.04, 1682.34], [53.09, 78.31] GW h under $p_{i}=0.01$ to [1760.32, 1885.78], [95.23, 427.16] GW h under $p_{i}=0.1$, respectively. These increased amounts are attributed to more availabilities of solar and wind energy resources when $\mathrm{p}_{\mathrm{i}}$ is at higher level.

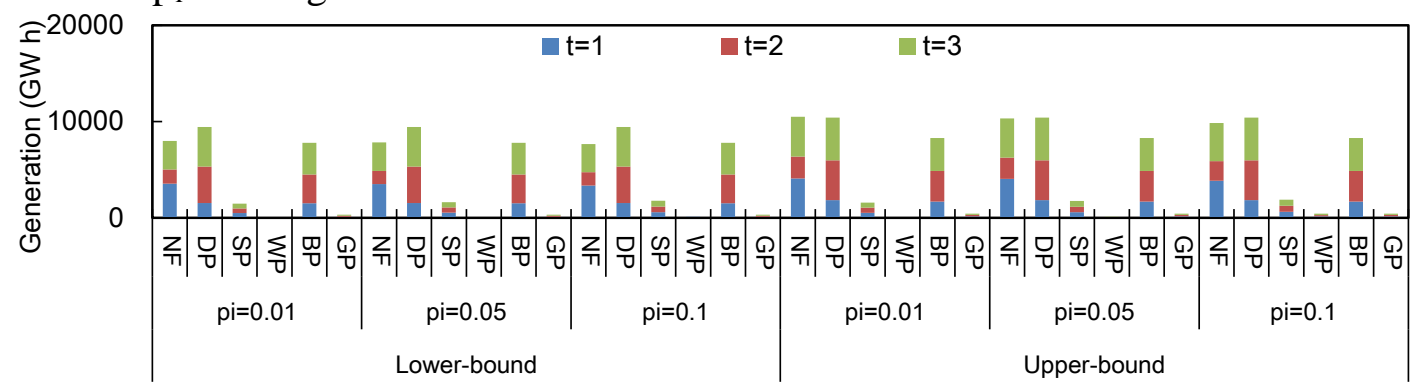

Fig. 1 Solutions of electricity generation under different pi levels.

\subsection{System cost and environmental pollution control}

Results of system cost and environmental effects are presented in Fig. 2. Results indicate that the amount of system cost and environmental pollution would decrease with the increase of $p_{i}$ level. The totaling system cost would amount to RMB $¥[106.16,161.70] \times 10^{8},[105.87,159.25] \times 10^{8}$ and [104.62, 158.73] $\times 10^{8}$ under $\mathrm{p}_{\mathrm{i}}=0.01,0.05$ and 0.1 , respectively. The related pollution emissions would respectively reach [20467, 26188], [20275, 25956] and [20067, 25347] tonnes under $\mathrm{p}_{\mathrm{i}}=0.01$, 0.05 and 0.1. Among them, the $\mathrm{SO}_{2}$-emission would be [688.14, 917.58], [679.69, 909.21] and [674.36, 893.15] tonnes under $\mathrm{p}_{\mathrm{i}}=0.01,0.05$ and 0.1 , respectively; the $\mathrm{NO}_{\mathrm{X}}$-emission would be [7847.76, 10195.94], [7764.91, 10096.63] and [7675.71, 9835.75] tonnes under $\mathrm{p}_{\mathrm{i}}=0.01,0.05$ and 0.1 , respectively; the $\mathrm{PM}_{10}$-emission would be [11931.53, 15074.42], [11827.70, 14948.38] and [11715.00, 14618.86] tonnes under $\mathrm{p}_{\mathrm{i}}=0.01,0.05$ and 0.1 , respectively. The decreased cost and pollutant emissions are associated with that more renewable energy resources are used for electricity generation, thus decrease environmental pollution emissions and cost for environmental treatment. Moreover, decisions at a lower $\mathrm{p}_{\mathrm{i}}$ level would decrease the risk of violating constraints, but with higher system cost and higher environmental effects; conversely, decisions at a higher $\mathrm{p}_{\mathrm{i}}$ level would return with lower system cost and lower environmental effects, but with a decreased system reliability.

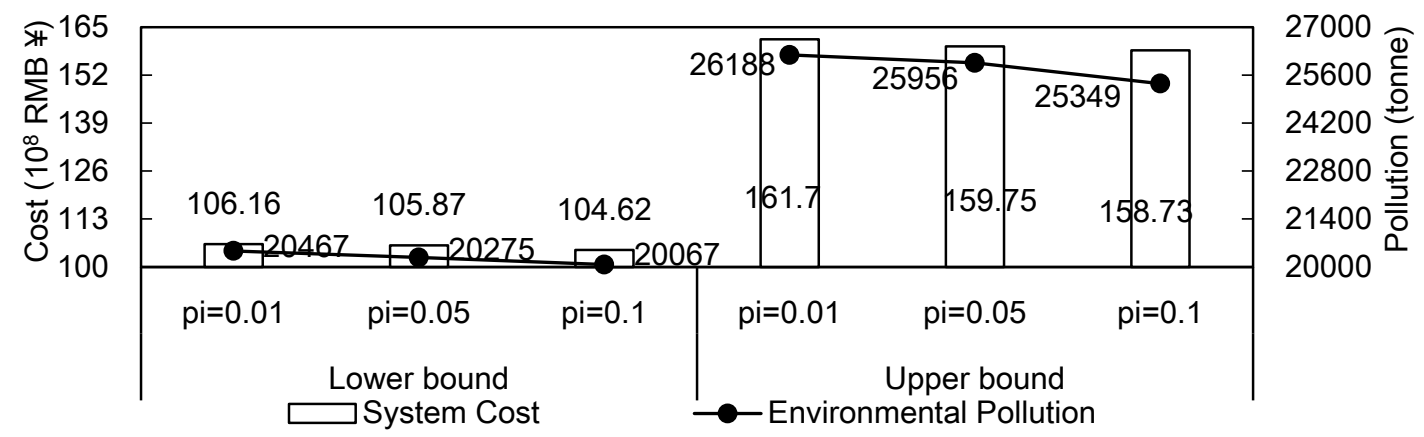

Fig. 2 Variations of system cost and environmental pollution under different pi levels.

\section{Summary}

An inexact stochastic bi-level programming model (ISBPM) is developed for planning electric power systems (EPS) in Fengtai District, Beijing, China. ISBPM can not only allow uncertainties expressed as interval and stochastic parameters, but also present a Leader-Follower process. With the aid of ISBPM, schemes related to electricity generation, system cost and environmental effects could be achieved. Results show that decisions with a higher constraint-violation risk would correspond to lower system cost and lower environmental effects but a decreased system reliability. 


\section{Acknowledgements}

This research was supported by the Program for China National Funds for Excellent Young Scientists (51222906), National Natural Science Foundation of China (41271540), New Century Excellent Talents in Universities of China (NCET-13-0791), and Fundamental Research Funds for the Central Universities.

\section{References}

[1].H.I. Calvete, C. Galé. Linear bilevel multi-follower programming with independent followers. Journal of Global Optimization. Vol. 39 (2007) No. 3, p. 409-417. 